\title{
Reservoir for Comet Material: Circumstellar Grains
}

\author{
Peter Hoppe
}

Received: 26 March 2007 / Accepted: 15 June 2007 / Published online: 16 August 2007

(C) Springer Science+Business Media B.V. 2007

\begin{abstract}
Primitive meteorites and interplanetary dust particles contain small quantities of dust grains with highly anomalous isotopic compositions. These grains formed in the winds of evolved stars and in the ejecta of stellar explosions, i.e., they represent a sample of circumstellar grains that can be analyzed with high precision in the laboratory. Such studies have provided a wealth of information on stellar evolution and nucleosynthesis, Galactic chemical evolution, grain growth in stellar environments, interstellar chemistry, and the inventory of stars that contributed dust to the Solar System. Among the identified circumstellar grains in primitive solar system matter are diamond, graphite, silicon carbide, silicon nitride, oxides, and silicates. Circumstellar grains have also been found in cometary matter. To date the available information on circumstellar grains in comets is limited, but extended studies of matter returned by the Stardust mission may help to overcome the existing gaps.
\end{abstract}

Keywords Solar system: formation · Comets: general - Meteors, meteoroids · Circumstellar matter · Dust, extinction · Nuclear reactions, nucleosynthesis, abundances

\section{Introduction}

Our Solar System formed from the collapse of an interstellar gas and dust cloud some 4.6 billion years ago. Only a small fraction of the solid matter that went into the making of the Solar System survived the earliest stages of Solar System formation. These relict samples ("presolar grains") are found in small quantities in primitive meteorites and interplanetary dust particles (IDPs). Presolar grains can be distinguished from solids that formed in the Solar System by their highly anomalous (with respect to average Solar System matter) isotopic compositions. They formed in the winds of evolved stars or in the ejecta of supernova explosions, i.e., they represent a sample of circumstellar grains that can be studied with a variety of high-precision analytical techniques in the laboratory. The study of presolar grains

P. Hoppe (两)

Max-Planck-Institute for Chemistry, Particle Chemistry Department, P.O. Box 3060, 55020 Mainz, Germany

e-mail: hoppe@mpch-mainz.mpg.de 
has opened a new window to astronomy. It complements astronomical observations and it permits us to obtain unique astrophysical information.

Comets are generally assumed to represent the most primitive solid matter in the Solar System. Recently, about $1 \mathrm{mg}$ of matter from the comet Wild 2 was brought to Earth by the Stardust mission (Brownlee et al. 2006). The preliminary examination of these samples indicates that the matter in Wild 2 is a mixture of presolar materials and of solids that formed in the Solar System itself. These analyses demonstrated that comet Wild 2 contains circumstellar grains, although with lower concentrations than expected (McKeegan et al. 2006). Since the study of Stardust samples is only at the beginning, the information on the presence and nature of presolar grains in comets is very limited (only one presolar grain was found to date). Thus, in this paper I will focus on presolar (circumstellar) grains found in meteorites and IDPs as a potential reservoir for cometary material. I will give a brief overview of the different types of presolar grains, and will describe their isotopic compositions and stellar sources. The topic of presolar grains was described in detail in several review papers (Anders and Zinner 1993; Ott 1993; Bernatowicz and Zinner 1997; Zinner 1998, 2004; Hoppe and Zinner 2000; Nittler 2003; Lodders and Amari 2005) and the interested reader is referred to these papers for more in-depth information and a full list of references.

\subsection{Presolar Minerals}

Table 1 lists the presolar minerals identified to date. Pictures of presolar grains are displayed in Fig. 1. Most abundant are the diamonds which are found with concentrations of

Table 1 Presolar minerals found in meteorites and interplanetary dust particles

\begin{tabular}{|c|c|c|c|c|}
\hline Mineral & Size $(\mu \mathrm{m})$ & $\begin{array}{l}\text { Abundance }^{\mathrm{a}} \\
(\mathrm{ppm})\end{array}$ & Stellar source & $\begin{array}{l}\text { Relative } \\
\text { contribution }^{\mathrm{b}}\end{array}$ \\
\hline Diamond & $\sim 0.0026$ & 1500 & Supernovae & $?$ \\
\hline Silicon carbide & $0.1-10$ & 30 & $\begin{array}{l}\text { AGB stars } \\
\text { J-type C stars? } \\
\text { Supernovae } \\
\text { Novae }\end{array}$ & $\begin{array}{l}>90 \% \\
<5 \% \\
1 \% \\
0.1 \%\end{array}$ \\
\hline Graphite & $1-10$ & 10 & $\begin{array}{l}\text { Supernovae } \\
\text { AGB stars } \\
\text { J-type C stars? } \\
\text { Novae }\end{array}$ & $\begin{array}{l}<80 \% \\
>10 \% \\
<10 \% \\
2 \%\end{array}$ \\
\hline Silicon nitride & $\sim 1$ & 0.002 & Supernovae & $100 \%$ \\
\hline $\begin{array}{l}\text { Oxides } \\
\left(\mathrm{MgAl}_{2} \mathrm{O}_{4}, \mathrm{Al}_{2} \mathrm{O}_{3},\right. \\
\left.\mathrm{CaAl}_{12} \mathrm{O}_{19}, \mathrm{TiO}_{2}\right)\end{array}$ & $0.1-5$ & 50 & $\begin{array}{l}\text { RGB/AGB stars } \\
\text { Supernovae }\end{array}$ & $\begin{array}{l}>90 \% \\
1 \%\end{array}$ \\
\hline Silicates & $0.1-1$ & $\begin{array}{l}200 \text { (Met.) } \\
800 \text { (IDPs) }\end{array}$ & $\begin{array}{l}\text { RGB/AGB stars } \\
\text { Supernovae }\end{array}$ & $\begin{array}{l}>90 \% \\
<10 \%\end{array}$ \\
\hline
\end{tabular}

\footnotetext{
${ }^{\text {a }}$ Reported maximum values from different meteorites are given.

${ }^{b}$ Note uncertainty about actual fraction of diamonds that are presolar and for the relative fractions of graphite grains that are attributed to AGB stars and supernovae.
} 
Fig. 1 Scanning electron microscopy images of circumstellar (presolar) dust found in meteorites. The circumstellar $\mathrm{SiC}, \mathrm{Al}_{2} \mathrm{O}_{3}$ and graphite grains were chemically extracted from meteorites; the circumstellar silicate grain was found in situ in the matrix of the Acfer 094 meteorite by ion imaging. The graphite photo courtesy of S. Amari

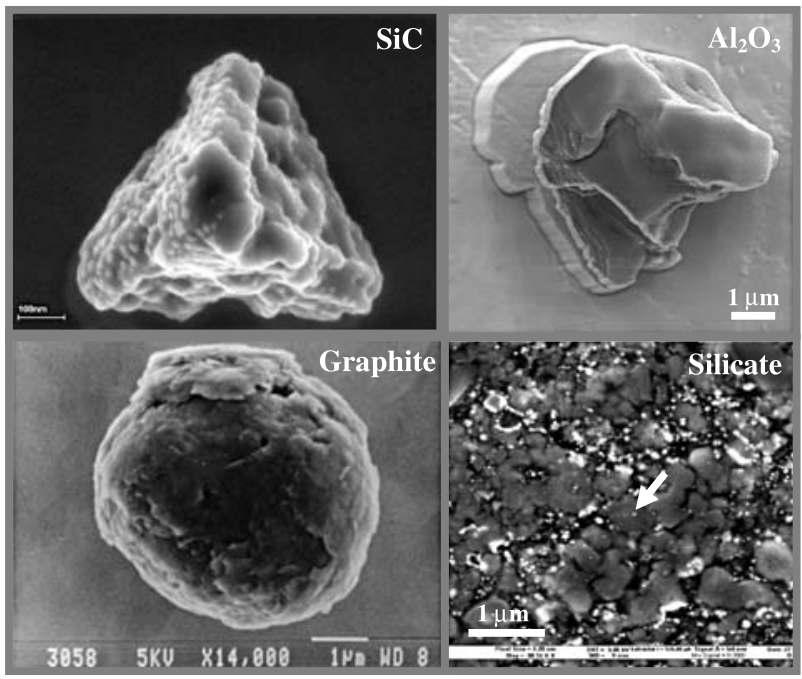

up to $1,500 \mathrm{ppm}$ (by weight) in the most primitive meteorites. What fraction of the diamonds is actually presolar is still a matter of debate. Diamonds are only $2-3 \mathrm{~nm}$ in size, too small for single-grain isotopic measurements. Bulk diamond samples exhibit isotopically normal (i.e., close-to-solar) C, but isotopically anomalous Xe (Lewis et al. 1987) and Te (Richter et al. 1998) which suggests that some (unknown) fraction of them is presolar. The next most abundant presolar minerals are silicates (variable composition) that are present with concentrations of up to several $100 \mathrm{ppm}$ and which were discovered only recently, first in IDPs (Messenger et al. 2003) and later also in meteorites (Nguyen and Zinner 2004; Mostefaoui and Hoppe 2004). Presolar refractory oxides, namely, spinel $\left(\mathrm{MgAl}_{2} \mathrm{O}_{4}\right)$, corundum $\left(\mathrm{Al}_{2} \mathrm{O}_{3}\right)$, hibonite $\left(\mathrm{CaAl}_{12} \mathrm{O}_{19}\right)$, and $\mathrm{TiO}_{2}$ (Hutcheon et al. 1994; Nittler et al. 1994; Choi et al. 1998), silicon carbide (SiC) (Bernatowicz et al. 1987), and graphite (Amari et al. 1990) are found with concentrations of more than $10 \mathrm{ppm}$. Like the diamonds, SiC and graphite can be separated from meteorites in almost pure form by chemical processing. Very rare are silicon nitride $\left(\mathrm{Si}_{3} \mathrm{~N}_{4}\right)$ grains (Nittler et al. 1995).

\subsection{Astrophysical Information}

Figure 2 shows the path of presolar grains from their stellar source to the laboratory. Their isotopic compositions are those in the atmosphere or in the ejecta of their stellar sources. These are determined from the compositions at stellar birth and the nucleosynthetic processes during stellar evolution. After passage through the interstellar medium (ISM) such grains became part of the molecular cloud from which our Solar System formed. A small fraction of the most refractory of those grains survived the period of enhanced temperatures in the solar nebula and was incorporated into growing planetesimals. In small planetary bodies, such as the asteroids and comets, they escaped subsequent destruction by planetary metamorphism. Finally, they are carried to the Earth by meteorites, IDPs, and sample return missions (Stardust). The refractory oxides and the carbonaceous grains can be separated by chemical and physical treatments which were invented by Ed Anders and co-workers at the University of Chicago (Amari et al. 1994); presolar silicates can be recognized by ion imaging techniques (see below). 


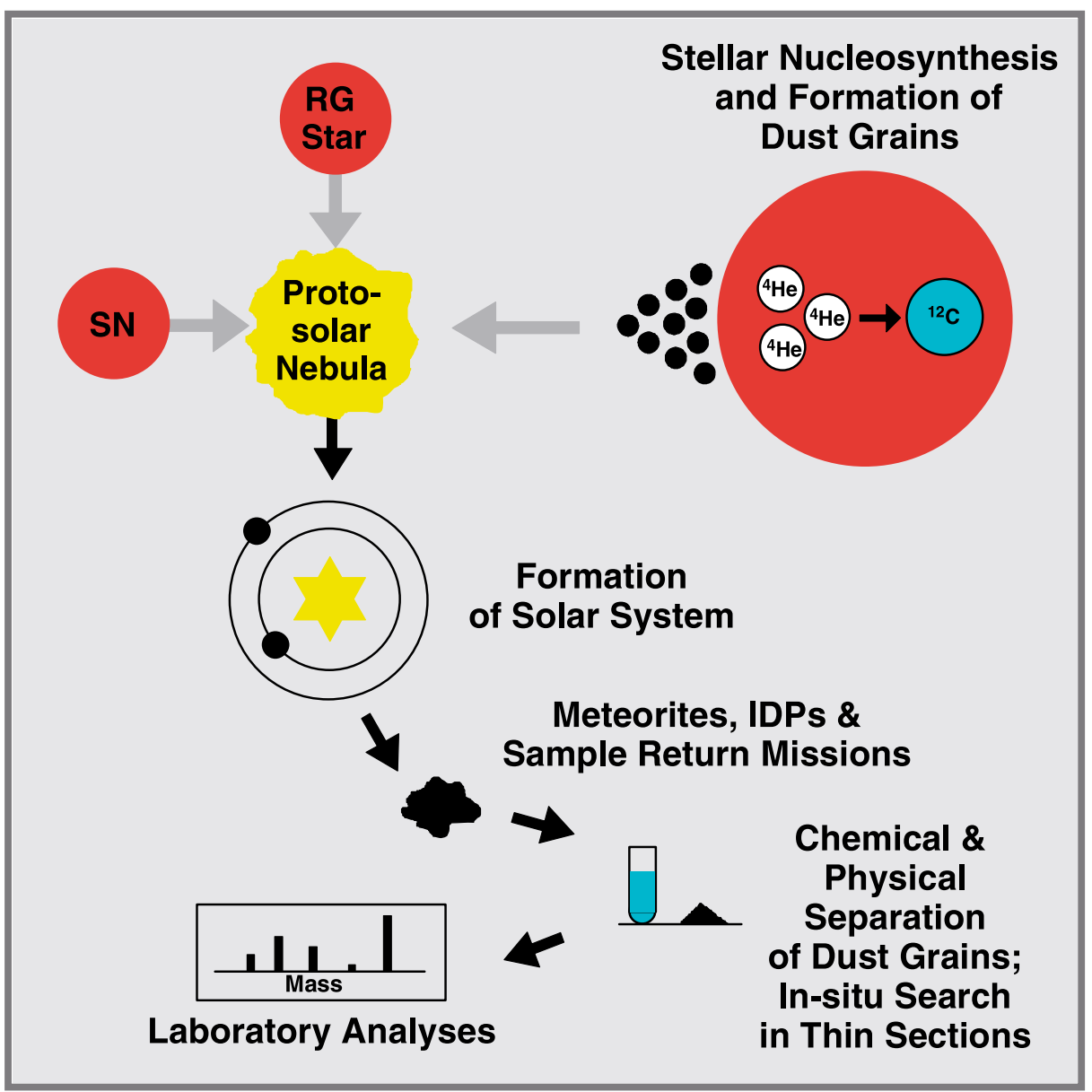

Fig. 2 The path of circumstellar grains from their stellar source to the laboratory. The grains can be either chemically and physically separated from primitive solar system materials or recognized in situ by isotope ion imaging

Important tools for the laboratory study of presolar grains are Secondary Ion Mass Spectrometry (SIMS) and Resonance Ionization Mass Spectrometry (RIMS) for isotope measurements of single grains, and Transmission Electron Microscopy (TEM) for investigations of structure and mineralogy. The laboratory study of presolar grains has provided a wealth of astrophysical information, such as on stellar nucleosynthesis and evolution, Galactic chemical evolution (GCE), mixing in supernova ejecta, grain formation in stellar environments, the chemistry in the ISM, and the types of stars that contributed dust to the Solar System.

\subsection{Astronomical Observations}

The types of presolar grains identified in primitive Solar System matter can be compared to astronomical observations of circumstellar dust (for an overview see Molster and Waters 2002). Most of the known presolar minerals are also observed in various stellar environments: Features of silicates (amorphous and crystalline) are seen in the spectra of young 


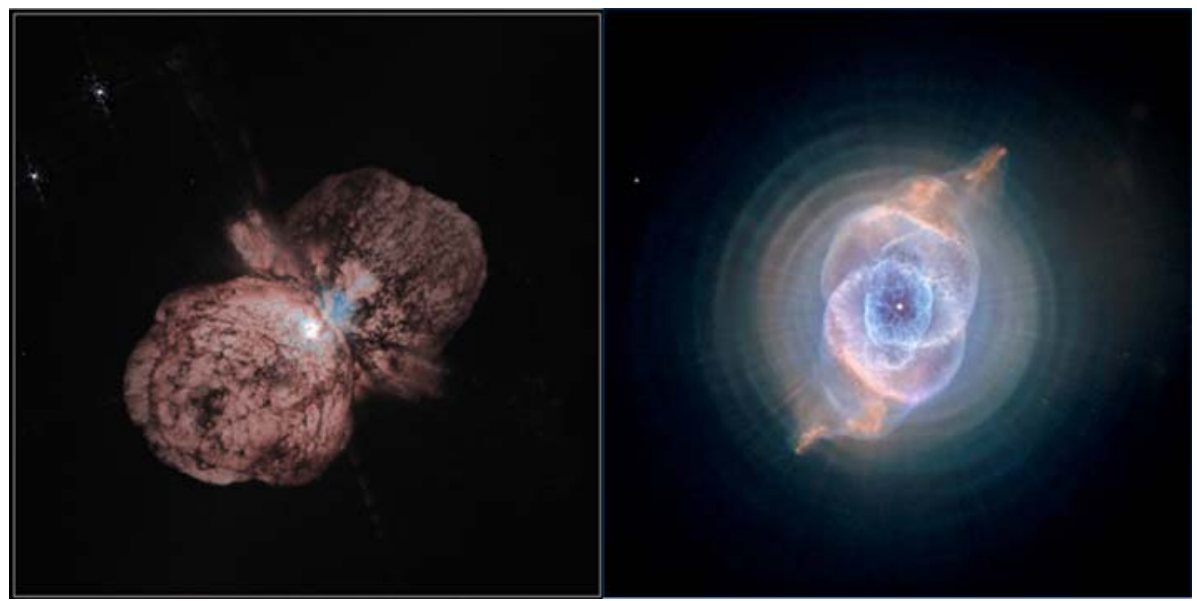

Fig. 3 Gas and dust emission from late-type stars: Hubble Space Telescope images of Eta Carinae (left) and the Cat's Eye Nebula (right). Eta Carinae is one of the most massive stars in our Galaxy $(\sim 100 \times$ heavier than our Sun) and suffered a giant outburst about 150 years ago. The evolved AGB star in the center of the Cat's Eye Nebula ejected its mass in a series of pulses at 1,500-year intervals. Photo credits: NASA/J. Morse (Eta Carinae), ESA, NASA, HEIC and the Hubble Heritage Team (STScI/AURA) (Cat's Eye Nebula)

stars, C- and O-rich asymptotic giant branch (AGB) stars, post-AGB stars, planetary nebulae, and massive stars. Refractory oxides are present in the atmosphere of O-rich AGB stars, carbonaceous dust around C-rich AGB stars, post-AGB stars, and planetary nebulae. Silicates and carbonaceous dust are also detected in the ISM. Other dust species such as $\mathrm{MgS}$ and $\mathrm{FeS}$ are observed in stellar spectra but have not been found as presolar grains yet. Figure 3 shows two examples of dust and gas emission from evolved stars. The most prolific suppliers of dust (O- and C-rich) to the ISM are AGB stars during different stages of their evolution (for an overview see Gail 2002). Other significant sources of dust are red supergiants, Wolf-Rayet stars and novae. Not much is known about dust from supernovae. However, some of the presolar grains found in meteorites and IDPs clearly come from supernovae (discussed later).

\section{Silicon Carbide}

Silicon carbide is the best-studied presolar mineral. Isotopic data are available not only for the major elements $\mathrm{C}$ and $\mathrm{Si}$ but also for a large number of minor elements that are contained in the $\mathrm{SiC}$ grains. This includes $\mathrm{N}, \mathrm{Mg}, \mathrm{Ca}$, Ti, the noble gases, and heavy refractory elements ( $\mathrm{Sr}, \mathrm{Zr}, \mathrm{Mo}, \mathrm{Ba}, \mathrm{Nd}, \mathrm{Sm}$, Dy). Based on the isotopic compositions of $\mathrm{C}, \mathrm{N}$, and $\mathrm{Si}$ and the abundance of radiogenic ${ }^{26} \mathrm{Mg}$ (from the decay of radioactive ${ }^{26} \mathrm{Al}$ which has a half life of 700,000 years), SiC was divided into six different populations (Hoppe and Ott 1997; Fig. 4): The mainstream grains which make up the majority of presolar $\mathrm{SiC}$ ( $>90 \%$ of all grains), type A\&B grains, including the sub-population of the rare nova grains, type $Y \& Z$ grains, and type $\mathrm{X}$ grains. There is strong evidence that the majority of presolar SiC grains formed in carbon stars, late-type AGB stars in which the $\mathrm{C} / \mathrm{O}$ ratio is $>1$ in the envelope:

(i) The distribution of $\mathrm{C}$-isotopic ratios in presolar $\mathrm{SiC}$ grains is similar to that measured for carbon stars. 
Fig. 4 C- and N-isotopic compositions of distinct presolar SiC populations. The dashed lines represent the solar ratios. Note that for the solar ${ }^{14} \mathrm{~N} /{ }^{15} \mathrm{~N}$ ratio the value inferred for Jupiter is taken (Owen et al. 2001). Data sources: Hoppe et al. (1994, 1996a, 1996b, 1997, 2000), Nittler et al. (1995), Gao and Nittler (1997), Huss et al. (1997), Amari et al. (2001a, 2001b, 2001c), and Nittler and Hoppe (2005)

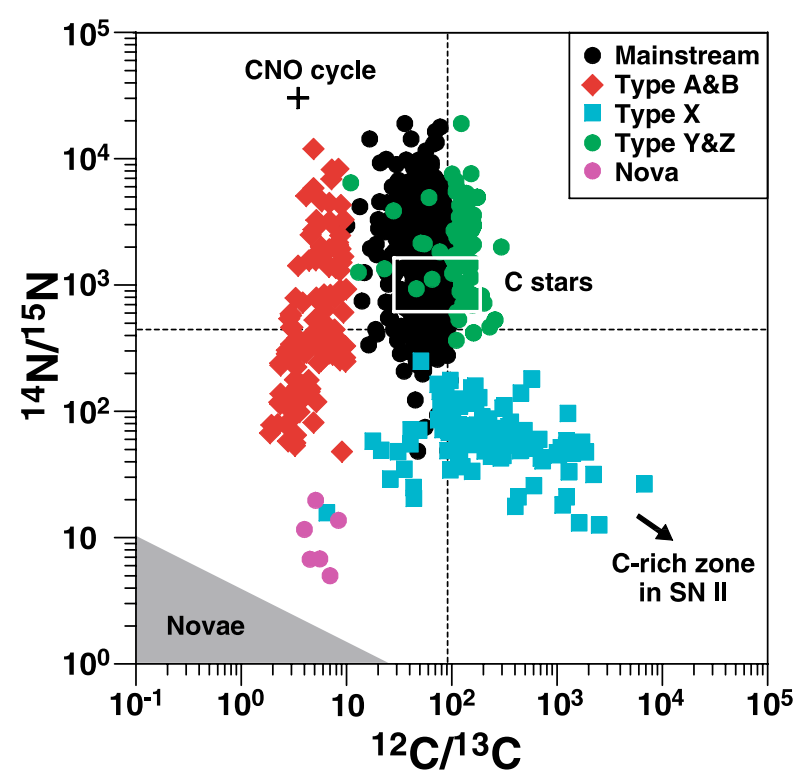

(ii) The AGB star-specific signature of s-process nucleosynthesis is seen in the isotopic patterns of intermediate-mass to heavy elements.

(iii) $\mathrm{SiC}$ is observed in stellar spectra (see Molster and Waters 2002).

(iv) Carbon stars are the most prolific injectors of dust into the ISM (see Gail 2002).

\subsection{Mainstream Grains}

The mainstream grains have ${ }^{12} \mathrm{C} /{ }^{13} \mathrm{C}$ ratios between 10 and 200 and ${ }^{14} \mathrm{~N} /{ }^{15} \mathrm{~N}$ ratios between 50 and 20,000 (Fig. 4). Most grains are characterized by enhanced ${ }^{13} \mathrm{C}$ and ${ }^{14} \mathrm{~N}$ compared to solar isotopic abundances. This is qualitatively the signature of $\mathrm{H}$ burning by the $\mathrm{CNO}$ cycle. The isotopic compositions of AGB star envelopes are changed during the star's evolution in three so-called dredge-up episodes when matter that experienced $\mathrm{H}$ and $\mathrm{He}$ burning and the s-process is mixed into the envelope. In the third dredge-up events the $\mathrm{C} / \mathrm{O}$ ratio eventually exceeds unity and such stars become carbon stars. Predicted ${ }^{12} \mathrm{C} /{ }^{13} \mathrm{C}$ ratios in carbon stars are in the range of most mainstream grains. Many mainstream grains, however, have higher ${ }^{14} \mathrm{~N} /{ }^{15} \mathrm{~N}$ ratios than predicted by the canonical models for carbon stars. Higher ${ }^{14} \mathrm{~N} /{ }^{15} \mathrm{~N}$ ratios can be achieved if "cool bottom processing" (CBP) is considered (Wasserburg et al. 1995). During CBP, which occurs subsequent to the first dredge-up, deep circulation currents transport matter from the non-burning bottom of the convective envelope down to regions where some $\mathrm{H}$ burning can occur. Other signatures of $\mathrm{H}$ and $\mathrm{He}$ burning in $\mathrm{SiC}$ mainstream grains are the former presence of radioactive ${ }^{26} \mathrm{Al}$ (Hoppe et al. 1994; Huss et al. 1997) and large isotopic overabundances of ${ }^{22} \mathrm{Ne}$ (e.g., Lewis et al. 1994; Heck et al. 2007).

The isotopic patterns of the intermediate-mass to heavy elements show the imprints of s-process (slow neutron capture) nucleosynthesis (e.g., Nicolussi et al. 1997, 1998; Savina et al. 2003; Marhas et al. 2007), in agreement with theoretical predictions for 1-3 $\mathrm{M}_{\odot} \mathrm{AGB}$ stars (Lugaro et al. 2003). It is interesting to note in this context that the detection of the radioactive s-process isotope ${ }^{99} \mathrm{Tc}$ (half life 200,000 years) in the spectra of AGB stars (Merrill 1952) has been the first direct evidence that the chemical elements are produced in the interior of stars. Fingerprints of ${ }^{99} \mathrm{Tc}$ have recently also been found in the isotopic pattern of Ru 
Fig. 5 Si-isotopic compositions of the distinct presolar $\mathrm{SiC}$ populations (except the $\mathrm{X}$ grains) given as permil deviation from the solar ${ }^{29} \mathrm{Si} /{ }^{28} \mathrm{Si}$ and ${ }^{30} \mathrm{Si} /{ }^{28} \mathrm{Si}$ ratios. Predictions for the evolution of the envelope of AGB stars with successive dredge-up of s-process $\mathrm{Si}$ is shown for comparison. Data sources: Hoppe et al. (1994), Gao and Nittler (1997), Amari et al. (2001c), and Nittler and Alexander (2003)

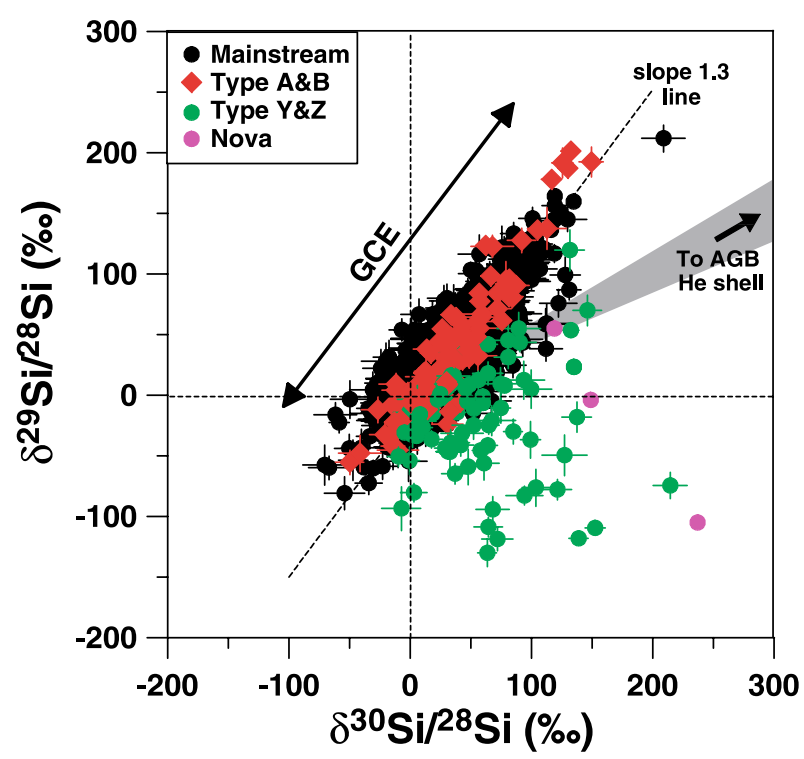

in $\mathrm{SiC}$ mainstream grains (Savina et al. 2004). The isotopic abundances of ${ }^{99} \mathrm{Ru}$ are higher than predicted for carbon stars, even if uncertainties in nuclear reaction rates are considered, but can be satisfactorily explained by contributions from now-extinct ${ }^{99} \mathrm{Tc}$.

Although $\mathrm{Si}$ is affected by the s-process, its isotopic composition is changed only marginally in the envelope of solar-metallicity AGB stars with progressive third dredge-up events. Most of the mainstream grains exhibit enrichments in the neutron-rich isotopes ${ }^{29} \mathrm{Si}$ and ${ }^{30} \mathrm{Si}$ of up to $200 \%$ and in a Si three-isotope representation the data plot along a line with slope $\sim 1.3$ (Fig. 5). The preferred interpretation of this line is that it reflects primarily the GCE, both in time and space, of the Si isotopes and represents a range of Si starting compositions of a large number of stars and not the effects of the stellar nucleosynthesis in the grain's parent stars (Alexander 1993; Gallino et al. 1994; Timmes and Clayton 1996; Lugaro et al. 1999).

\subsection{Type $\mathrm{Y}$ and Z Grains}

The abundance of type $\mathrm{Y}$ and $\mathrm{Z}$ grains increases with decreasing grain size, from about $1 \%$ for grains $>2 \mu \mathrm{m}$ to about 5-7\% for grains of $0.5 \mu \mathrm{m}$ (Zinner et al. 2007). While ${ }^{12} \mathrm{C} /{ }^{13} \mathrm{C}$ ratios in type $\mathrm{Y}$ grains (Amari et al. 2001a) are higher than in mainstream grains, in type $\mathrm{Z}$ grains they are similar to those of mainstream grains (Hoppe et al. 1997; Nittler and Alexander 2003; Zinner et al. 2007) (Fig. 4). The type Y and Z grains have $\mathrm{N}$-isotopic compositions (Fig. 4) and ${ }^{26} \mathrm{Al}$ abundances that are indistinguishable from those of the mainstream grains. In the Si three-isotope representation the type $\mathrm{Y}$ and $\mathrm{Z}$ grains plot to the ${ }^{30} \mathrm{Si}$-rich side of the Si mainstream line (Fig. 5). This and the observed large excesses in ${ }^{50} \mathrm{Ti}$ are compatible with an origin in low-metallicity (1/3 to $1 / 2$ solar) AGB stars.

\subsection{Type A and B Grains}

The minor type $\mathrm{A}$ and $\mathrm{B}$ grains have ${ }^{12} \mathrm{C} /{ }^{13} \mathrm{C}$ ratios $<10$ (Amari et al. 2001b). Many of these grains have lower than solar ${ }^{14} \mathrm{~N} /{ }^{15} \mathrm{~N}$ ratios (Fig. 4). Their Si isotopic ratios are compatible 
Fig. $6 \mathrm{Si}$-isotopic compositions of presolar $\mathrm{SiC}$ grains of type $\mathrm{X}$. The range of $\mathrm{SiC}$ mainstream grains is shown for comparison. Data sources: Nittler et al. (1995), Hoppe et al. (2000), Besmehn (2001), Besmehn and Hoppe (2003), and Nittler and Alexander (2003)

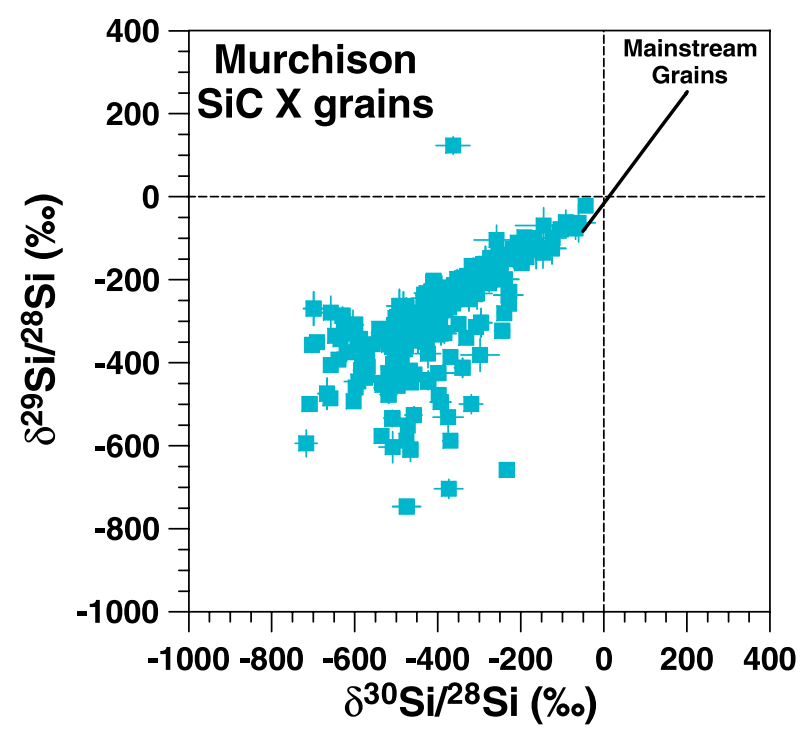

with those of the mainstream grains (Fig. 5) except for the sub-population of the nova grains (Amari et al. 2001c; Nittler and Hoppe 2005) which plot to the ${ }^{30} \mathrm{Si}$-rich side of the Si mainstream line. Besides novae for grains with very large ${ }^{15} \mathrm{~N}$ excesses, J-type carbon stars and born-again AGB stars have been proposed as potential stellar sources for the type A and B grains. Both types of stars are poorly understood at present.

\subsection{Type X Grains}

$\mathrm{SiC} \mathrm{X}$ grains are very rare (about $1 \%$ of all presolar $\mathrm{SiC}$ grains). By ion imaging in the ion microprobe (Nittler et al. 1997; Hoppe et al. 2000) X grains can be efficiently identified and a large isotope data base exists for X grains (e.g., Amari et al. 1992; Nittler et al. 1996; Hoppe et al. 2000; Hoppe and Besmehn 2002; Besmehn and Hoppe 2003; Nittler and Alexander 2003). The $X$ grains have ${ }^{12} \mathrm{C} /{ }^{13} \mathrm{C}$ ratios between 6 and 7,000 and ${ }^{14} \mathrm{~N} /{ }^{15} \mathrm{~N}$ ratios between $\sim 10$ and 250 (Fig. 4). All grains have isotopically heavy $\mathrm{N}$ and most of them have higher than solar ${ }^{12} \mathrm{C} /{ }^{13} \mathrm{C}$ ratios. It is interesting to note that the in situ isotope measurements of matter ejected by comet Halley yielded C-rich particles with isotopically light $\mathrm{C}$ having ${ }^{12} \mathrm{C} /{ }^{13} \mathrm{C}$ ratios of up to $\sim 5,000$ (Jessberger and Kissel 1991) which falls in the range of the SiC X grains. The Si-isotopic compositions of X grains are displayed in Fig. 6. The X grains exhibit depletions in the neutron-rich isotopes ${ }^{29} \mathrm{Si}$ and ${ }^{30} \mathrm{Si}$, or alternatively speaking, enrichments in ${ }^{28} \mathrm{Si}$. This signature can be explained only by a contribution of matter that experienced advanced nuclear burning stages. This makes supernova explosions the most likely sources of the X grains. Supernovae (SNe) of Type II are preferred (Hoppe et al. 2000), but SNe of Type Ia cannot fully be ruled out (Clayton et al. 1997). Another characteristic feature of $\mathrm{X}$ grains are very high ${ }^{26} \mathrm{Al} /{ }^{27} \mathrm{Al}$ ratios of up to 0.6 , much higher than those observed in grains from AGB stars.

Many of the $\mathrm{X}$ grains show large excesses in ${ }^{44} \mathrm{Ca}$ and ${ }^{49} \mathrm{Ti}$ which are best explained by the decay of radioactive ${ }^{44} \mathrm{Ti}$ (half life 60 years) and ${ }^{49} \mathrm{~V}$ (half life 330 days). These isotopes are produced in the innermost Ni- and Si-rich zones of Type II SNe (Woosley and Weaver 1995) and their presence is considered a proof for a SN origin of the X grains. Since ${ }^{49} \mathrm{Ti}$ excesses are positively correlated with $\mathrm{V} / \mathrm{Ti}$ ratios, ${ }^{49} \mathrm{~V}$ must have been alive when the 
$\mathrm{X}$ grains condensed. This constrains the time of grain formation to several months after SN explosion (Hoppe and Besmehn 2002), which is consistent with astronomical observations of dust formation in SN1987a (Wooden 1997).

One $\mathrm{X}$ grain with ${ }^{12} \mathrm{C} /{ }^{13} \mathrm{C}=6$ and ${ }^{14} \mathrm{~N} /{ }^{15} \mathrm{~N}=16$ plots in the range generally attributed to nova grains. Its Si-isotopic signature, ${ }^{26} \mathrm{Al} /{ }^{27} \mathrm{Al}$ ratio, and ${ }^{44} \mathrm{Ca}$ excess, however, points to a supernova origin (Nittler and Hoppe 2005). This suggests coupled synthesis of ${ }^{13} \mathrm{C}$ and ${ }^{15} \mathrm{~N}$ not only in nova explosions but also in Type II SNe.

In grains from $\mathrm{SNe}$, the isotopic patterns of heavy elements are expected to show the effects of the r-process (rapid neutron capture) nucleosynthesis. This, however, could not be confirmed. Measurements of Mo in X grains by RIMS revealed large excesses in ${ }^{95} \mathrm{Mo}$ and ${ }^{97} \mathrm{Mo}$ (Pellin et al. 1999). In the classical r-process the largest excesses are expected for ${ }^{100}$ Mo. The observed pattern can be explained with a neutron burst in shocked He-rich matter (Meyer et al. 2000). The occurrence of this kind of neutron burst is a natural consequence of Type II SN explosions (Rauscher et al. 2002). This gives further support that X grains are from Type II SNe, and not from Type Ia, because this kind of neutron burst is not expected to occur in Type Ia SNe.

Presolar $\mathrm{Si}_{3} \mathrm{~N}_{4}$ grains show the same isotopic signatures as $\mathrm{X}$ grains: Low ${ }^{29} \mathrm{Si} /{ }^{28} \mathrm{Si}$ ${ }^{30} \mathrm{Si} /{ }^{28} \mathrm{Si}$ and ${ }^{14} \mathrm{~N} /{ }^{15} \mathrm{~N}$ ratios, and high ${ }^{26} \mathrm{Al} /{ }^{27} \mathrm{Al}$ ratios (Nittler et al. 1995). This points to a close relationship between these two types of grains, making Type II SNe the most likely sources of presolar $\mathrm{Si}_{3} \mathrm{~N}_{4}$.

\section{Graphite}

Presolar graphite grains are round, typically $>1 \mu \mathrm{m}$ in size, and have a range in density $\left(1.6-2.2 \mathrm{~g} / \mathrm{cm}^{3}\right.$ ) (Amari et al. 1990). Many grains contain small internal grains, mostly TiC (Bernatowicz et al. 1991), but also Mo- and Zr-rich carbides (Bernatowicz et al. 1996) and kamacite, cohenite, and iron grains (Bernatowicz et al. 1999; Croat et al. 2003). TiC, kamacite, cohenite, and iron grains have been observed in grains of likely $\mathrm{SN}$ origin (see below).

The range of ${ }^{12} \mathrm{C} /{ }^{13} \mathrm{C}$ ratios of graphite grains $(2-7,000)$ is similar to that seen in presolar $\mathrm{SiC}$; the distribution of ${ }^{12} \mathrm{C} /{ }^{13} \mathrm{C}$ ratios, however, is clearly different (Fig. 7). Most graphite grains have higher than solar ${ }^{12} \mathrm{C} /{ }^{13} \mathrm{C}$ ratios, as similarly observed for the $\mathrm{SiC} \mathrm{X}$ grains. Most of the low-density graphite grains appear to come from $\mathrm{SNe}$, as indicated by excesses in ${ }^{15} \mathrm{~N},{ }^{18} \mathrm{O},{ }^{28} \mathrm{Si}$, and high ${ }^{26} \mathrm{Al} /{ }^{27} \mathrm{Al}$ ratios (Amari et al. 1995a; Travaglio et al. 1999). Strong support for a SN origin also comes from evidence for now-extinct ${ }^{44} \mathrm{Ti}$ (Nittler et al. 1996) and ${ }^{41} \mathrm{Ca}$ (half life 105,000 years; Amari et al. 1996) observed in a few grains. Internal $\mathrm{TiC}$ grains show large excesses in ${ }^{49} \mathrm{Ti}$ (Stadermann et al. 2005a), as similarly observed for $\mathrm{X}$ grains, suggestive of decay of ${ }^{49} \mathrm{~V}$. Surprisingly, TiC sub-grains are rich in O (Stadermann et al. 2005a). They exhibit large excesses in ${ }^{18} \mathrm{O}$ and the ${ }^{18} \mathrm{O} /{ }^{16} \mathrm{O}$ ratios are even more extreme than that of the host graphite.

Some (unknown) fraction of high-density graphite grains appear to originate from AGB stars. This is indicated by the presence of internal refractory carbide grains (especially TiC), most of which have significant concentrations of $\mathrm{Zr}$, Mo, and $\mathrm{Ru}$ in solid solution (Croat et al. 2005), and by the s-process isotopic patterns of Mo and $\mathrm{Zr}$ in several graphite grains (Nicolussi et al. 1998), as similarly observed in SiC mainstream grains. Further support for AGB stars comes from $\mathrm{Kr}$ isotope data of graphite bulk samples that show the AGB starspecific s-process pattern (Amari et al. 1995b).

A small fraction of the graphite grains probably comes from novae. Presolar graphite is the carrier of the noble gas component $\mathrm{Ne}-\mathrm{E}(\mathrm{L})$, which consists of almost pure ${ }^{22} \mathrm{Ne}$ 
Fig. 7 Histograms of ${ }^{12} \mathrm{C} /{ }^{13} \mathrm{C}$ ratios of presolar graphite and $\mathrm{SiC}$ grains. Data for graphite are from Hoppe et al. (1995), those for $\mathrm{SiC}$ from Hoppe et al. (1994, 1996a)

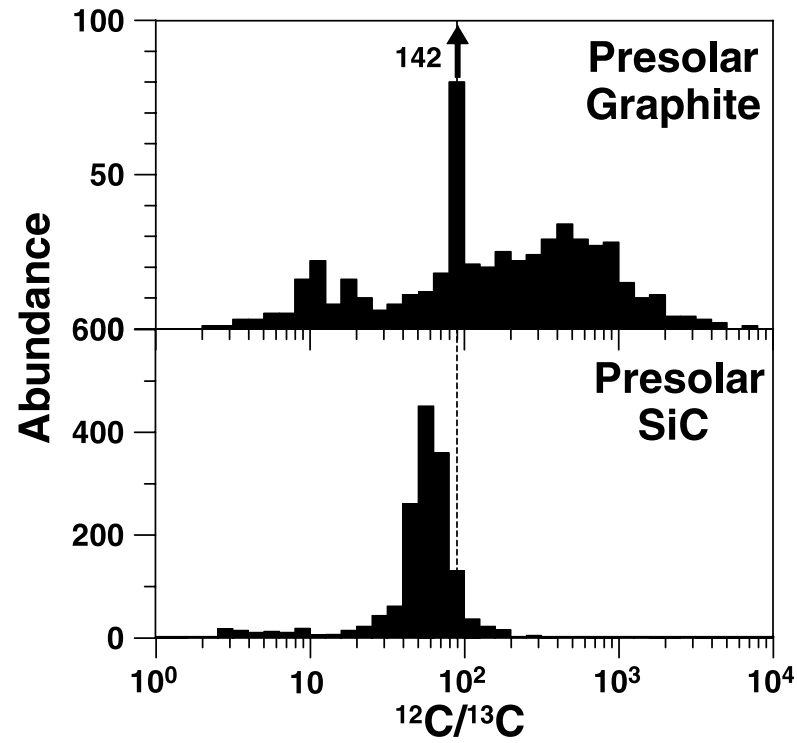

Fig. 8 O-isotopic compositions of presolar oxide and silicate grains. The solar ratios are given by the dashed lines. The four isotope groups of O-rich presolar dust are indicated by the grey-shaded ellipses.

Data sources: Nittler et al. (1994, 1997, 1998), Messenger et al. (2003, 2005), Mostefaoui and Hoppe (2004), Nguyen and Zinner (2004), Hoppe et al. (2005), Stadermann et al. (2005b), Zinner et al. (2005), Floss et al. (2006), Marhas et al. (2006), Yada et al. (2006), Nguyen et al. (2007), and Vollmer et al. (2007)

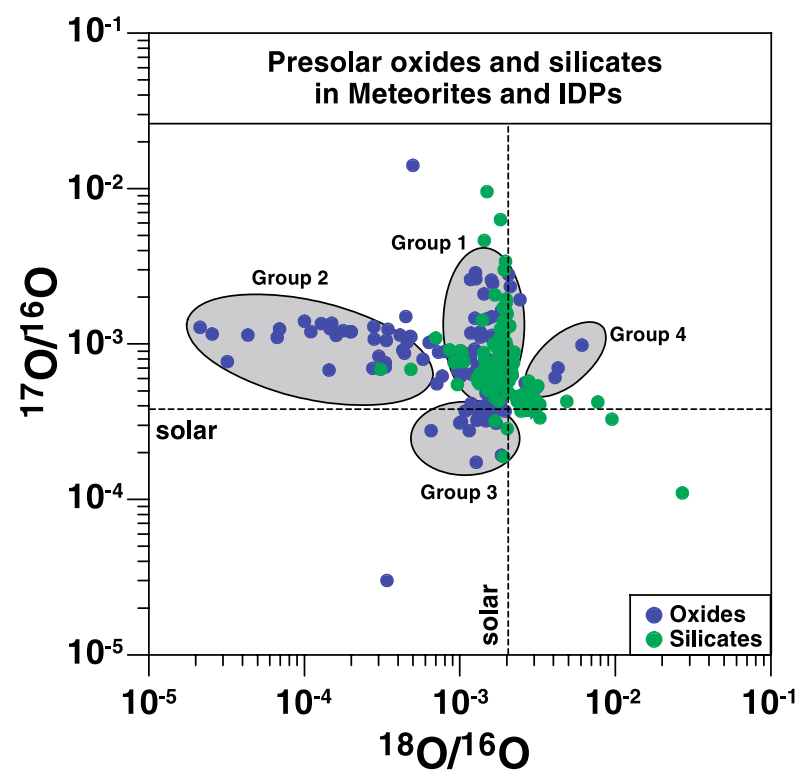

(Amari et al. 1990). The low ${ }^{20} \mathrm{Ne} /{ }^{22} \mathrm{Ne}$ ratio of $\mathrm{Ne}-\mathrm{E}(\mathrm{L})$ has been attributed to an origin in novae where ${ }^{22} \mathrm{Ne}$ is produced by the decay of radioactive ${ }^{22} \mathrm{Na}$ (half life 2.6 years) (Clayton 1975). Noble gas measurements on single graphite grains by laser heating revealed three grains with low ${ }^{20} \mathrm{Ne} /{ }^{22} \mathrm{Ne}$ and low ${ }^{12} \mathrm{C} /{ }^{13} \mathrm{C}$ ratios compatible with an origin in novae (Nichols et al. 1992,1994; Heck et al. 2007). However, as $\mathrm{SNe}$ also produce ${ }^{22} \mathrm{Na}$ and as a $\mathrm{SN} \mathrm{SiC} \mathrm{X}$ grain with the $\mathrm{C}$ - and $\mathrm{N}$-isotopic signature of nova grains was found (see Sect. 2.4), a SN origin for the three graphite grains with low ${ }^{20} \mathrm{Ne} /{ }^{22} \mathrm{Ne}$ and low ${ }^{12} \mathrm{C} /{ }^{13} \mathrm{C}$ ratios cannot be ruled out. 


\section{Oxides}

Based on their O-isotopic compositions, the oxide grains (corundum, spinel, hibonite) were divided into four groups (Nittler et al. 1997; see Fig. 8). Comparisons between the grain data and spectroscopic observations as well as stellar model predictions suggest RGB and AGB stars as the most likely stellar sources for the majority of the grains. Group 1 grains have enhanced ${ }^{17} \mathrm{O} /{ }^{16} \mathrm{O}$ ratios and close-to-solar or slightly lower than solar ${ }^{18} \mathrm{O} /{ }^{16} \mathrm{O}$ ratios. This is the signature of core $\mathrm{H}$ burning in low- to intermediate-mass $\left(1-2.5 \mathrm{M}_{\odot}\right)$ stars followed by dredge-up of core material into the envelope. While the ${ }^{17} \mathrm{O} /{ }^{16} \mathrm{O}$ ratios are mostly determined by stellar mass, the varying ${ }^{18} \mathrm{O} /{ }^{16} \mathrm{O}$ ratios reflect different metallicities of the parent stars (e.g., Boothroyd and Sackmann 1999). Group 2 grains show higher than solar ${ }^{17} \mathrm{O} /{ }^{16} \mathrm{O}$ ratios and strong depletions in ${ }^{18} \mathrm{O}$. This signature has been explained by CBP (Wasserburg et al. 1995; Nollett et al. 2003) in low-mass $\left(<1.65 \mathrm{M}_{\odot}\right)$ stars during the AGB phase. Group 3 grains have lower than solar ${ }^{17} \mathrm{O} /{ }^{16} \mathrm{O}$ and ${ }^{18} \mathrm{O} /{ }^{16} \mathrm{O}$ ratios, pointing to low-mass stars with lower than solar metallicity as stellar sources. Group 4 grains exhibit enrichments in ${ }^{17} \mathrm{O}$ and ${ }^{18} \mathrm{O}$. Proposed stellar sources for these grains include highmetallicity AGB stars and Type II SNe for grains with large ${ }^{18} \mathrm{O}$ excesses. The majority of $\mathrm{O}$-rich grains from Type II SNe is expected to show strong enrichments in ${ }^{16} \mathrm{O}$. To date only one grain with this O-isotopic signature was observed (Fig. 8, Nittler et al. 1998). Many grains from all four groups carry radiogenic ${ }^{26} \mathrm{Mg}$ from the decay of now extinct ${ }^{26} \mathrm{Al}$. Because ${ }^{26} \mathrm{Al}$ is produced by shell $\mathrm{H}$ burning during the thermal pulsing AGB phase, grains with evidence for ${ }^{26} \mathrm{Al}$ must have formed during the AGB phase, those without during the preceding RGB phase.

\section{Silicates}

While presolar carbonaceous and oxide grains can be separated by harsh chemical treatments, this does not hold for silicates. The absence of silicates among the known reservoir of presolar grains in meteorites has been an unsatisfactory fact for a long time because silicates are the major constituent of O-rich dust around stars (see Sect. 1.3). Only the invention of the NanoSIMS ion microprobe made the discovery of presolar silicates possible. The first silicates were found by NanoSIMS ion imaging on an interplanetary dust particle by Messenger et al. (2003). In this technique a focused $\mathrm{Cs}^{+}$ion beam $(<100 \mathrm{~nm})$ is rastered over areas

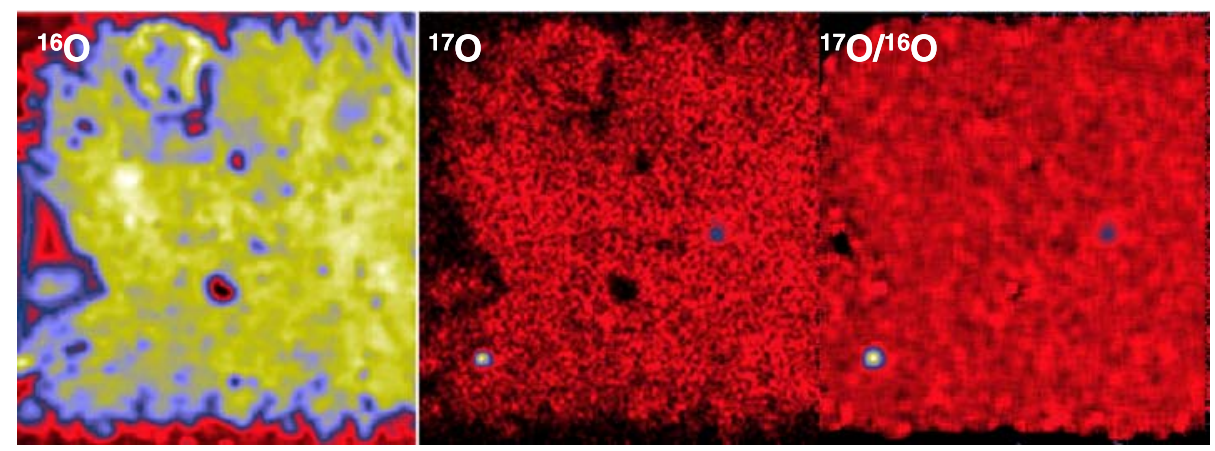

Fig. 9 NanoSIMS ion images of ${ }^{16} \mathrm{O},{ }^{17} \mathrm{O}$, and ${ }^{17} \mathrm{O} /{ }^{16} \mathrm{O}$ in an area $9 \times 9 \mu \mathrm{m}^{2}$ in size in the matrix of the Acfer 094 meteorite. Two presolar silicate grains can be recognized by strong enrichments in ${ }^{17} \mathrm{O}$ (from Hoppe et al. 2005) 
typically some $100 \mu \mathrm{m}^{2}$ in size and simultaneous ion images of selected isotopes (e.g., ${ }^{16} \mathrm{O}$, ${ }^{17} \mathrm{O},{ }^{18} \mathrm{O}$ ) are acquired to search for local isotopic anomalies. An example is shown in Fig. 9, where two presolar silicate grains, about $300 \mathrm{~nm}$ in size, are seen as hotspots in the ${ }^{17} \mathrm{O}$ and ${ }^{17} \mathrm{O} /{ }^{16} \mathrm{O}$ ion images of an area in the matrix of the Acfer 094 meteorite (Hoppe et al. 2005).

Most presolar silicates are enriched in ${ }^{17} \mathrm{O}$ and have close-to-solar or slightly lower than solar ${ }^{18} \mathrm{O} /{ }^{16} \mathrm{O}$ ratio (Fig. 8) as it is similarly observed for the presolar oxides, i.e., they belong to Group 1. Grains of Group 2 are apparently rare. This, however, could be an observational bias from in situ ion imaging because small contributions from the surrounding meteorite matrix to the ion signal from the presolar grains would increase ${ }^{18} \mathrm{O} /{ }^{16} \mathrm{O}$ ratios considerably (Nguyen et al. 2007). As it was concluded for the presolar oxide grains, most presolar silicate grains apparently formed in the winds of RGB and AGB stars and contributions from $\mathrm{SNe}$ are rare. Only one grain, the one with the lowest ${ }^{17} \mathrm{O} /{ }^{16} \mathrm{O}$ and highest ${ }^{18} \mathrm{O} /{ }^{16} \mathrm{O}$ ratios (Fig. 8), clearly can be attributed to a $\mathrm{SN}$ source. This is based not only on its unusual O-isotopic signature but also on its $\mathrm{Si}$-isotopic signature which is similar to that of the SiC X grains (Messenger et al. 2005).

Information on the detailed mineralogy of the presolar silicates is limited to date. This is because the presolar silicates are not easily accessible to Transmission Electron Microscopy investigations. Among the 10 grains analyzed to date are four so-called GEMS
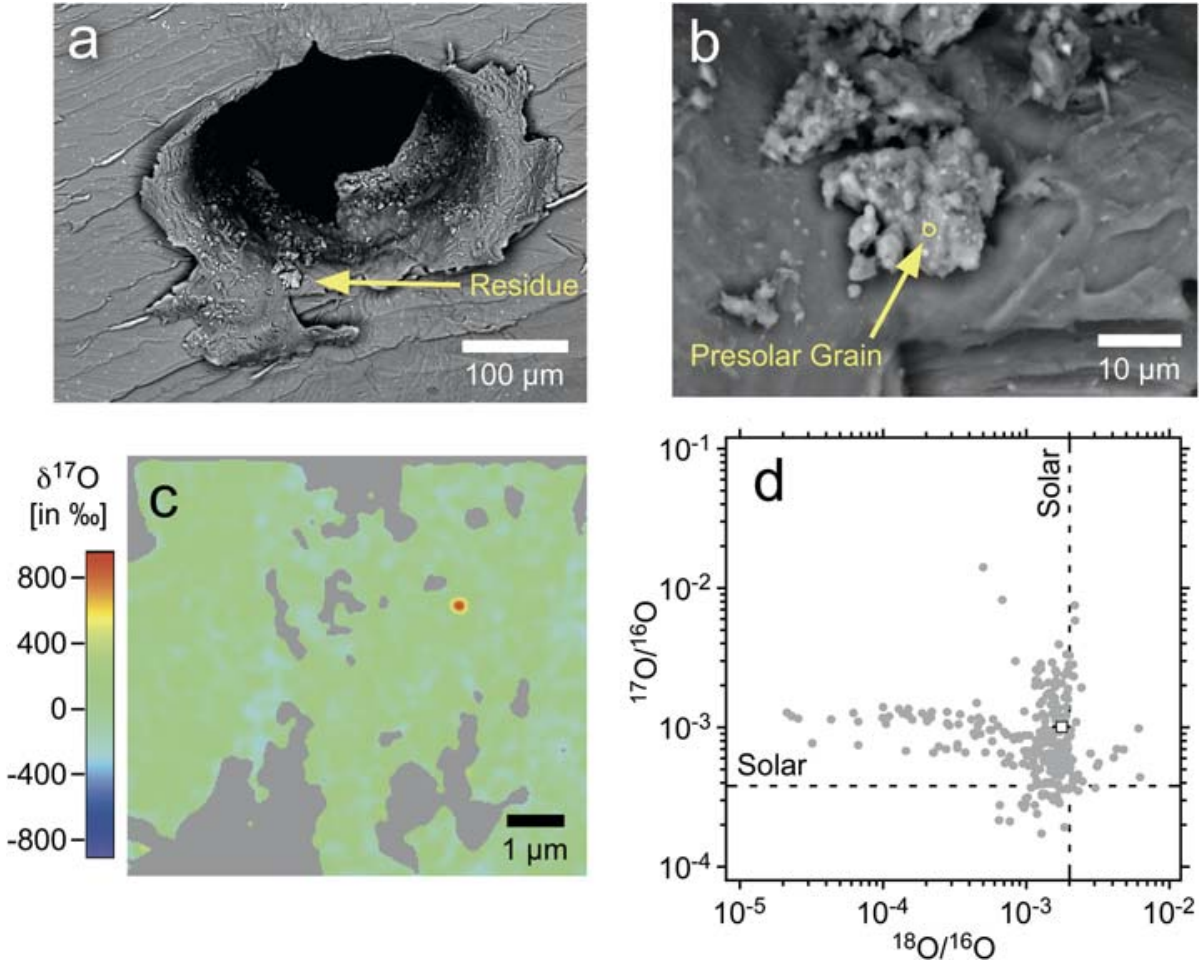

Fig. 10 The only circumstellar grain from the Stardust mission identified to date. (a) SEM image of an impact crater in the Al foil where the circumstellar grain was found. (b) Projectile residue in crater lip. (c) $\delta^{17} \mathrm{O}$ map acquired with the NanoSIMS. The circumstellar grain is clearly seen as a red hotspot. (d) O-isotopic ratios of the circumstellar grain (open square) and literature data for circumstellar oxide grains from meteorites. Figure taken from McKeegan et al. (2006) 
("glass with embedded metal and sulfides"), two amorphous Fe-rich silicates, two olivines $\left((\mathrm{Mg}, \mathrm{Fe})_{2} \mathrm{SiO}_{4}\right)$, one $\mathrm{MgSiO}_{3}$ with a perovskite-like structure, and a composite grain with an Al-rich core (probably $\mathrm{Al}_{2} \mathrm{O}_{3}$ ) which is surrounded by a silicate mantle (e.g., Messenger et al., 2003, 2005; Floss et al. 2006; Vollmer et al., 2006, 2007).

\section{Implications for Comets}

Most comets formed in the outer fringe of our Solar System. They incorporated primitive solar system matter which remained largely unaffected since the time the comets formed. In view of the results obtained for primitive meteorites, presolar (circumstellar) grains can thus be expected to be important ingredients of comets. In fact, in situ studies onboard the Giotto spacecraft of matter from comet Halley indicated solid C-rich particles with isotopically light C (Jessberger and Kissel 1991), a signature observed in many presolar graphite and SiC SN grains. Matter from comet Wild 2, captured by the Stardust spacecraft and returned to Earth recently, can be studied in the laboratory with the best available instrumentation. Only a single circumstellar grain could be identified during the first analysis phase (Fig. 10; McKeegan et al. 2006; Stadermann et al. 2007), which represents some $20 \mathrm{ppm}$ of the analyzed cometary matter. The detailed mineralogy of the grain is not known but it is likely to be an oxide or silicate. It is characterized by a strong enrichment in ${ }^{17} \mathrm{O}$ and slight depletion in ${ }^{18} \mathrm{O}$ with respect to solar isotopic abundances with ${ }^{17} \mathrm{O} /{ }^{16} \mathrm{O} \sim 0.001$ and ${ }^{18} \mathrm{O} /{ }^{16} \mathrm{O}$ $\sim 0.0018$, i.e., it belongs to Group 1 of O-rich presolar dust (cf. Fig. 8) and likely formed in the wind of a RGB/AGB star. It should be emphasized that the detection efficiency for presolar grains in Wild 2 material is clearly less than $100 \%$ and that the true abundance of circumstellar grains could be much higher. The analysis of Stardust material is just at the beginning and it is hoped that extended laboratory studies of cometary matter will reveal more and possibly new presolar minerals not known from meteorites. This will help to get a detailed look at the starting material from which the comets formed.

Acknowledgements I thank the International Space Science Institute at Bern, Switzerland, for organizing the workshop "Origin and Evolution of Comet Nuclei" where this paper was presented.

\section{References}

C.M.O.D. Alexander, Geochim. Cosmochim. Acta 57, 2869-2888 (1993)

S. Amari, E. Anders, A. Virag, E. Zinner, Nature 345, 238-240 (1990)

S. Amari, P. Hoppe, E. Zinner, R.S. Lewis, Astrophys. J. 394, L43-L46 (1992)

S. Amari, R.S. Lewis, E. Anders, Geochim. Cosmochim. Acta 58, 459-470 (1994)

S. Amari, E. Zinner, R.S. Lewis, Astrophys. J. 447, L147-L150 (1995a)

S. Amari, R.S. Lewis, E. Anders, Geochim. Cosmochim. Acta 53, 1411-1426 (1995b)

S. Amari, E. Zinner, R.S. Lewis, Astrophys. J. 470, L101-L104 (1996)

S. Amari, L.R. Nittler, E. Zinner, R. Gallino, M. Lugaro, R.S. Lewis, Astrophys. J. 546, 248-266 (2001a)

S. Amari, L.R. Nittler, E. Zinner, K. Lodders, R.S. Lewis, Astrophys. J. 559, 463-483 (2001b)

S. Amari, X. Gao, L.R. Nittler, E. Zinner, Astrophys. J. 551, 1065-1072 (2001c)

E. Anders, E. Zinner, Meteoritics 28, 490-514 (1993)

T.J. Bernatowicz, E. Zinner (eds.), Astrophysical Implications of the Laboratory Study of Presolar Materials, New York. AIP Conf. Proc., vol. 402, p. 750 (1997)

T.J. Bernatowicz, G. Fraundorf, T. Ming, E. Anders, B. Wopenka, E. Zinner, P. Fraundorf, Nature 330, 728730 (1987)

T.J. Bernatowicz, S. Amari, E. Zinner, R.S. Lewis, Astrophys. J. 373, L73-L76 (1991)

T.J. Bernatowicz, R. Cowsik, P.C. Gibbons, K. Lodders, B. Fegley Jr., S. Amari, R.S. Lewis, Astrophys. J. 472, 760-782 (1996) 
T.J. Bernatowicz, J. Bradley, S. Amari, S. Messenger, R.S. Lewis, Lunar Planet. Sci. 30 (1999). Abstract 1392 (CD-ROM)

A. Besmehn, Ph.D. thesis, Johannes-Gutenberg-Universität, Mainz (2001)

A. Besmehn, P. Hoppe, Geochim. Cosmochim. Acta 67, 4693-4703 (2003)

A.I. Boothroyd, I.-J. Sackmann, Astrophys. J. 510, 232-250 (1999)

D.E. Brownlee et al., Science 314, 1711-1716 (2006)

B.-G. Choi, G.R. Huss, G.J. Wasserburg, R. Gallino, Science 282, 1284-1289 (1998)

D.D. Clayton, Nature 257, 36-37 (1975)

D.D. Clayton, W.D. Arnett, J. Kane, B.S. Meyer, Astrophys. J. 486, 824-834 (1997)

T.K. Croat, T.J. Bernatowicz, S. Amari, S. Messenger, F.J. Stadermann, Geochim. Cosmochim. Acta 67, 4705-4725 (2003)

T.K. Croat, F.J. Stadermann, T.J. Bernatowicz, Astrophys. J. 631, 976-987 (2005)

C. Floss, F.J. Stadermann, J.P. Bradley, Z.R. Dai, S. Bajt, G. Graham, A.S. Lea, Geochim. Cosmochim. Acta 70, 2371-2399 (2006)

H.-P. Gail, in Astromineralogy, ed. by T. Henning (Springer, New York, 2002), pp. 55-120

X. Gao, L.R. Nittler, Lunar Planet. Sci. 28, 393-394 (1997)

R. Gallino, C.M. Raiteri, M. Busso, F. Matteucci, Astrophys. J. 430, 858-869 (1994)

P.R. Heck, K.K. Marhas, P. Hoppe, R. Gallino, H. Baur, R. Wieler, Astrophys. J. 656, 1208-1222 (2007)

P. Hoppe, A. Besmehn, Astrophys. J. 576, L69-L72 (2002)

P. Hoppe, U. Ott, in Astrophysical Implications of the Laboratory Study of Presolar Materials, ed. by T.J. Bernatowicz, E. Zinner (AIP, New York, 1997), pp. 27-58

P. Hoppe, E. Zinner, J. Geophys. Res.-Space Phys. 105, 10371-10385 (2000)

P. Hoppe, S. Amari, E. Zinner, T. Ireland, R.S. Lewis, Astrophys. J. 430, 870-890 (1994)

P. Hoppe, S. Amari, E. Zinner, R.S. Lewis, Geochim. Cosmochim. Acta 59, 4029-4056 (1995)

P. Hoppe, R. Strebel, P. Eberhardt, S. Amari, R.S. Lewis, Geochim. Cosmochim. Acta 60, 883-907 (1996a)

P. Hoppe, T.A. Kocher, R. Strebel, P. Eberhardt, S. Amari, R.S. Lewis, Lunar Planet. Sci. 27, 561-562 (1996b)

P. Hoppe, P. Annen, R. Strebel, P. Eberhardt, R. Gallino, M. Lugaro, S. Amari, R.S. Lewis, Astrophys. J. 487, L101-L104 (1997)

P. Hoppe, R. Strebel, P. Eberhardt, S. Amari, R.S. Lewis, Meteorit. Planet. Sci. 35, 1157-1176 (2000)

P. Hoppe, S. Mostefaoui, T. Stephan, Lunar Planet. Sci. 36 (2005). Abstr. \#1301 (CD-ROM)

G.R. Huss, I.D. Hutcheon, G.J. Wasserburg, Geochim. Cosmochim. Acta 61, 5117-5148 (1997)

I.D. Hutcheon, G.R. Huss, A.J. Fahey, G.J. Wasserburg, Astrophys. J. 425, L97-L100 (1994)

E. Jessberger, J. Kissel, in Comets in the Post-Halley Era, ed. by R.L. Newburn, M. Neugebauer, J. Rahe (Kluwer Academic, Dordrecht, 1991), pp. 1075-1092

R.S. Lewis, M. Tang, J.F. Wacker, E. Anders, E. Steel, Nature 326, 160-162 (1987)

R.S. Lewis, S. Amari, E. Anders, Geochim. Cosmochim. Acta 58, 471-494 (1994)

K. Lodders, S. Amari, Chemie der Erde 65, 93-166 (2005)

M. Lugaro, E. Zinner, R. Gallino, S. Amari, Astrophys. J. 527, 369-394 (1999)

M. Lugaro, A.M. Davis, R. Gallino, M.J. Pellin, O. Straniero, F. Käppeler, Astrophys. J. 593, 486-508 (2003)

K.K. Marhas, P. Hoppe, F.J. Stadermann, C. Floss, A.S. Lea, Lunar Planet. Sci. 37 (2006). Abstr. \#1959 (CD-ROM)

K.K. Marhas, P. Hoppe, U. Ott, Meteorit. Planet. Sci. (2007, in press)

K.D. McKeegan et al., Science 314, 1724-1728 (2006)

P.W. Merrill, Astrophys. J. 116, 21-26 (1952)

S. Messenger, L.P. Keller, F. Stadermann, R.M. Walker, E. Zinner, Science 300, 105-108 (2003)

S. Messenger, L.P. Keller, D.S. Lauretta, Science 309, 737-741 (2005)

B.S. Meyer, D.D. Clayton, L.-S. The, Astrophys. J. 540, L49-L52 (2000)

F.J. Molster, L.B.F.M. Waters, in Astromineralogy, ed. by T. Henning (Springer, New York, 2002), pp. 121170

S. Mostefaoui, P. Hoppe, Astrophys. J. 613, L149-L152 (2004)

A.N. Nguyen, E. Zinner, Science 303, 1496-1499 (2004)

A.N. Nguyen, F.J. Stadermann, E. Zinner, R.M. Stroud, C.M.O.D. Alexander, L.R. Nittler, Astrophys. J. 656, 1223-1240 (2007)

R.H. Nichols Jr., C.M. Hohenberg, P. Hoppe, S. Amari, R.S. Lewis, Lunar Planet. Sci. 23, 989-990 (1992)

R.H. Nichols Jr., K. Kehm, R. Brazzle, S. Amari, C.M. Hohenberg, R.S. Lewis, Meteoritics 29, 510-511 (1994)

G.K. Nicolussi, A.M. Davis, M.J. Pellin, R.S. Lewis, R.N. Clayton, S. Amari, Science 277, 1281-1283 (1997)

G.K. Nicolussi, M.J. Pellin, R.S. Lewis, A.M. Davis, S. Amari, R.N. Clayton, Geochim. Cosmochim. Acta 62, 1093-1104 (1998) 
L.R. Nittler, Earth Planet. Sci. Lett. 209, 259-273 (2003)

L.R. Nittler, C.M.O.D. Alexander, Geochim. Cosmochim. Acta 67, 4961-4980 (2003)

L.R. Nittler, P. Hoppe, Astrophys. J. 631, L89-L92 (2005)

L.R. Nittler, C.M.O.D. Alexander, X. Gao, R.M. Walker, E.K. Zinner, Nature 370, 443-446 (1994)

L.R. Nittler, P. Hoppe, C.M.O.D. Alexander, S. Amari, P. Eberhardt, X. Gao, R.S. Lewis, R. Strebel, R.M. Walker, E. Zinner, Astrophys. J. 453, L25-L28 (1995)

L.R. Nittler, S. Amari, E. Zinner, S.E. Woosley, R.S. Lewis, Astrophys. J. 462, L31-L34 (1996)

L.R. Nittler, C.M.O.D. Alexander, X. Gao, R.M. Walker, E. Zinner, Astrophys. J. 483, 475-495 (1997)

L.R. Nittler, C.M.O.D. Alexander, J. Wang, X. Gao, Nature 393, 222 (1998)

K.M. Nollett, M. Busso, G.J. Wasserburg, Astrophys. J. 582, 1036-1058 (2003)

U. Ott, Nature 364, 25-33 (1993)

T. Owen, P.R. Mahaffy, H.B. Niemann, S. Atreya, M. Wong, Astrophys. J. 553, L77-L79 (2001)

M.J. Pellin, A.M. Davis, R.S. Lewis, S. Amari, R.N. Clayton, Lunar Planet. Sci. 30 (1999). Abstr. \#1969 (CD-ROM)

T. Rauscher, A. Heger, R.D. Hoffman, S.E. Woosley, Astrophys. J. 576, 323-348 (2002)

S. Richter, U. Ott, F. Begemann, Nature 391, 261-263 (1998)

M.R. Savina, A.M. Davis, C.E. Tripa, M.J. Pellin, R.N. Clayton, R.S. Lewis, S. Amari, R. Gallino, M. Lugaro, Geochim. Cosmochim. Acta 67, 3201-3214 (2003)

M.R. Savina, A.M. Davis, C.E. Tripa, M.J. Pellin, R. Gallino, R.S. Lewis, S. Amari, Science 303, 649-652 (2004)

F.J. Stadermann, T.K. Croat, T.J. Bernatowicz, S. Amari, S. Messenger, R.M. Walker, E. Zinner, Geochim. Cosmochim. Acta 69, 177-188 (2005a)

F.J. Stadermann, C. Floss, P.A. Bland, E.P. Vicenzi, D. Rost, Lunar Planet. Sci. 36 (2005b). Abstr. \#2004 (CD-ROM)

F.J. Stadermann, P. Hoppe, C. Floss, P.R. Heck, F. Hörz, J. Huth, A.T. Kearsley, J. Leitner, K.K. Marhas, K.D. McKeegan, T. Stephan, Meteorit. Planet. Sci. (2007, in press)

F.X. Timmes, D.D. Clayton, Astrophys. J. 472, 723-741 (1996)

C. Travaglio, R. Gallino, S. Amari, E. Zinner, S. Woosley, R.S. Lewis, Astrophys. J. 510, 325-354 (1999)

C. Vollmer, P. Hoppe, F.F. Brenker, R.M. Stroud, C. Holzapfel, Meteorit. Planet. Sci. 41, A184 (2006)

C. Vollmer, P. Hoppe, F. Brenker, C. Holzapfel, Lunar Planet. Sci. 38 (2007). Abstr. \#1262 (CD-ROM)

G.J. Wasserburg, A.I. Boothroyd, I.-J. Sackmann, Astrophys. J. 447, L37-L40 (1995)

D. Wooden, in Astrophysical Implications of the Laboratory Study of Presolar Materials, ed. by T.J. Bernatowicz, E. Zinner (AIP, New York, 1997), pp. 317-378

S.E. Woosley, T.A. Weaver, Astrophys. J. Suppl. 101, 181-235 (1995)

T. Yada, F.J. Stadermann, C. Floss, E. Zinner, T. Nakamura, T. Noguchi, A.S. Lea, Lunar Planet. Sci. 37 (2006). Abstr. \#1470 (CD-ROM)

E. Zinner, Ann. Rev. Earth Planet. Sci. 26, 147-188 (1998)

E. Zinner, in Treatise in Geochemistry, ed. by K.K. Turekian, H.D. Holland, A.M. Davis (Elsevier, Oxford/San Diego, 2004), pp. 17-39

E. Zinner, L.R. Nittler, P. Hoppe, R. Gallino, O. Straniero, C.M.O.D. Alexander, R.S. Lewis, Geochim. Cosmochim. Acta 69, 4149-4165 (2005)

E. Zinner, S. Amari, R. Guinness, C. Jennings, A.F. Mertz, A.N. Nguyen, R. Gallino, P. Hoppe, M. Lugaro, L.R. Nittler, R.S. Lewis, Geochim. Cosmochim. Acta (2007, in press) 